\title{
Spectrally Resolved Specular Reflections of Thermal Phonons from Atomically Rough Surfaces
}

\author{
Navaneetha K. Ravichandran \\ Department of Physics, Boston College, Chestnut Hill, Massachusetts 02467, USA \\ Hang Zhang \\ Institute of Engineering Thermophysics, Chinese Academy of Sciences, Beijing 100190, China \\ Austin J. Minnich* \\ Division of Engineering and Applied Science, California Institute of Technology, \\ Pasadena, California 91125, USA
}

(Received 22 March 2018; revised manuscript received 13 September 2018; published 5 October 2018)

\begin{abstract}
The reflection of waves from rough surfaces is a fundamental process that plays a role in diverse fields such as optics, acoustics, and seismology. While a quantitative understanding of the reflection process has long been established for many types of waves, the precise manner in which thermal phonons of specific wavelengths reflect from atomically rough surfaces remains unclear owing to limited control over terahertzfrequency phonon generation and detection. Knowledge of these processes is critical for many applications, however, and is particularly important for recent attempts to create novel materials by coherently interfering thermal phonons. Here, we report measurements of a key property for these efforts, the phononwavelength-dependent specularity parameter, which describes the probability of specular reflections of thermal phonons at a surface. Our experiments show evidence of specular surface reflections of terahertz thermal phonons in our samples around room temperature and indicate a sensitivity of these reflections to surface imperfections on the scale of just 2-3 atomic planes. Our work demonstrates a general route to probe the microscopic interactions of thermal phonons with surfaces that are typically inaccessible with traditional experiments.
\end{abstract}

DOI: 10.1103/PhysRevX.8.041004

Subject Areas: Condensed Matter Physics,

Energy Research, Materials Science

\section{INTRODUCTION}

The manner in which waves reflect from rough surfaces is a fundamental problem that plays an important role in fields ranging from optics to acoustics [1-3]. The problem is equally important for thermal phonons of terahertz (THz) frequencies that carry heat in crystals [4]. Rough surfaces resist heat flow by disrupting the propagation of lattice waves along the transport direction, and in nanostructures this resistance can dominate over other sources of resistance [5-8]. Whether phonons reflect specularly from a surface and, therefore, preserve their phase is also critical for coherent manipulation of heat, which is now a topic of intense interest [9-12].

\footnotetext{
*aminnich@caltech.edu
}

Published by the American Physical Society under the terms of the Creative Commons Attribution 4.0 International license. Further distribution of this work must maintain attribution to the author(s) and the published article's title, journal citation, and DOI.
Despite the importance of this process, the manner in which an incident thermal phonon reflects from a surface remains poorly understood. The outcome of a phonon reflection is mathematically described by the specularity parameter $p_{\lambda}$, which is the probability of specular, phasepreserving reflections of a given phonon mode with wavelength $\lambda$ from a surface. However, determining $p_{\lambda}$ for a given surface remains extremely challenging, either computationally or experimentally. Unlike for light, e.g., sources and detectors of $\mathrm{THz}$ phonons of specific vibrational modes are not readily available.

Efforts to determine the specularity parameter have proceeded by both theory and experiment over many decades. Ziman [13] originally derived a simple expression for the specularity parameter for a random rough surface as a function of the root-mean-square (rms) roughness amplitude of the surface $\eta$ and the wavelength of the phonon $\lambda$ under the assumption of an infinite correlation length and normal incidence of phonons on the surface as $p_{\lambda}^{\text {Ziman }}=\exp \left(-16 \pi^{2} \eta^{2} / \lambda^{2}\right)$. More recently, Maznev [14] derived the specularity parameter for a rough surface with a 
finite Gaussian correlation length by solving the elastic wave equation for an isotropic medium and showed that Ziman's formula will likely overestimate the thermal resistance due to boundary scattering. Green's functions $[15,16]$, molecular dynamics [17], and wave-packet simulations $[18,19]$ have also been used to study the effect of surface roughness on phonon scattering at the boundaries of complex nanostructure geometries.

Experimentally, Pohl and co-workers originally investigated the nature of phonon reflections from surfaces over a temperature range of a few millikelvin to room temperature, observing qualitative evidence for partially specular scattering of phonons below $10 \mathrm{~K}[20,21]$. More recently, Gelda et al. [22] reported specular reflections of longitudinal phonons with frequencies less than $0.1 \mathrm{THz}$ at room temperature in Si membranes and obtained good agreement with Ziman's specularity model. Heron et al. [23] also reported agreement with Ziman's theory for acoustic phonons by measuring the thermal conductance of $\mathrm{Si}$ nanowires around $1 \mathrm{~K}$. On the other hand, Hertzberg et al. [24] reported that Ziman's theory underpredicts diffuse scattering of sub-THz phonons at the surfaces of Si nanosheets. Thus, the recent experimental literature is contradictory on the validity of Ziman's theory even for sub-THz acoustic phonons $(\lambda \gtrsim 100 \AA)$, and so it is unclear if Ziman's theory applies for thermal phonons with frequencies of a few THz $(\lambda \lesssim 100 \AA)$.

A number of other recent works with varied experimental approaches measure thermal conductivities of thin films and nanowires and conclude that thermal phonons undergo predominantly diffuse surface reflections [25-36]. Their conclusions remain tentative, however, because in many experiments the observable quantity, thermal conductivity, is averaged over the broad blackbody phonon spectrum, complicating efforts to resolve the specularity parameter as a function of the phonon wavelength for thermal phonons.

Some of these prior works use thickness-dependent thermal-conductivity measurements on thin films to partially alleviate this difficulty [25-27,30-36]. While, in principle, thickness-dependent measurements could provide new spectral information on phonon surface reflections, extracting the specularity parameter quantitatively requires identical surface-roughness statistics for all thin films with varying thickness, which is not confirmed in these earlier works. Furthermore, apart from Ref. [31], an $a b$ initio baseline for the thermal conductivity in the specular and diffuse limits in the experimental configuration, which is crucial to extract the specularity parameter, was not available for prior works. These limitations also impede efforts to validate the models described earlier. Thus, a firm theoretical or experimental determination of the manner in which thermal phonons reflect from a rough surface is lacking in the literature.

Here, we report an approach that overcomes these challenges, allowing us to obtain the first measurements of the specularity parameter versus phonon wavelength for thermal phonons on individual atomically rough silicon membranes. First, we partially eliminate the undesirable phonon spectral averaging in thermal measurements by observing quasiballistic thermal transport rather than heat diffusion only. Second, we extract the wavelengthdependent specularity parameter for each thin-film sample separately, thus eliminating the effects of sample-to-sample variation of surface-roughness statistics on the final result. Finally, we interpret our measurements using numerical solutions of the phonon Boltzmann transport equation with $a b$ initio phonon properties as input, providing an accurate baseline for the specular and diffuse limits. We find evidence for specular reflections of thermal phonons even around room temperature for samples with wafer-scale roughness, while predominantly diffuse reflections are observed for samples exhibiting surface imperfections on the scale of just 2-3 atomic planes as determined from transmission electron microscopy. Our work demonstrates how microscopic information about the surface reflections of $\mathrm{THz}$ thermal phonons can be extracted from macroscopic thermal measurements by an interpretation of the experimental observables with an $a b$ initio description of phonon transport.

\section{THEORY}

The problem we seek to solve is to extract the specularity parameter resolved over the thermal phonon spectrum from observables in a thermal-transport experiment. Generally, the specularity parameter could depend on the wave vector and polarization. However, typical thermal measurements occur over areas much larger than the coherence length of surface roughness, leading to an observable that obscures directional information. Even if the length scale of thermal measurements is reduced to the order of magnitude of the coherence length of surface roughness, the number of unknown parameters to be determined for the directiondependent specularity parameter vastly exceeds the number of constraints that could be obtained from the experimental observations. Therefore, in the following theory, we mathematically define the quantity to be extracted from experiments as the directionally averaged specularity parameter versus phonon wavelength.

Even with this reduction in the number of unknown parameters, extracting the spectrally resolved specularity parameter is difficult. In most thermal experiments, the observable consists of measurements of thermal conductivity of a sample in which surface reflections contribute to the thermal resistance. Recovering the wavelengthdependent specularity parameter from these data alone is a fundamentally ill-posed problem, because the observables are additionally averaged over the broad phonon spectrum.

Prior works show that this undesirable phonon spectral averaging can be partially eliminated by observing quasiballistic thermal transport rather than heat diffusion only 
[29,37-45]. Intuitively, a lack of scattering over the heating length scale implies that the averaging process does not occur at least for some thermal phonons, yielding new spectral information about the phonon interactions. For concreteness, consider the transient-grating (TG) experiment we employ in this work [Fig. 1(a)], where a sinusoidal temperature profile is initially created in the in-plane direction of a sample by the interference of two laser pulses and the subsequent thermal decay is measured by a probe laser. The timescales in our TG experiment are on the order of nanoseconds or longer, and so the ultrafast relaxation processes of the electron-hole pairs and hot phonons are not observed; rather, the experiment measures the transient thermal decay associated with phonon transport. If the grating period is very long compared to the mean free paths (MFPs) of phonons within the sample, microscopic information about phonon surface reflections is obscured due to averaging by intrinsic phonon-phonon scattering. As the grating period is decreased, phonons with MFPs longer than the grating period transport heat ballistically and carry less heat compared to the prediction by Fourier's law of heat diffusion [46,47]. This difference between the heat carried by phonons in the heat diffusion and ballistic conduction regimes provides new spectral information about the phonon interactions. Particularly in thin films, this quasiballistic heat-conduction regime gives new spectral information about phonon-boundary interactions if the intrinsic phonon-phonon scattering rates are known.

Mathematically, the observable in the TG experiment on a thin film, the time constant of the thermal decay, can be expressed as $\tau^{-1}=\kappa(q) / C q^{2}$, where $q=2 \pi / \delta$ is the grating wave vector corresponding to a grating period $\delta$, $C$ is the volumetric specific heat, and $\kappa(q)$ is the apparent thermal conductivity, given by [47]

(a)

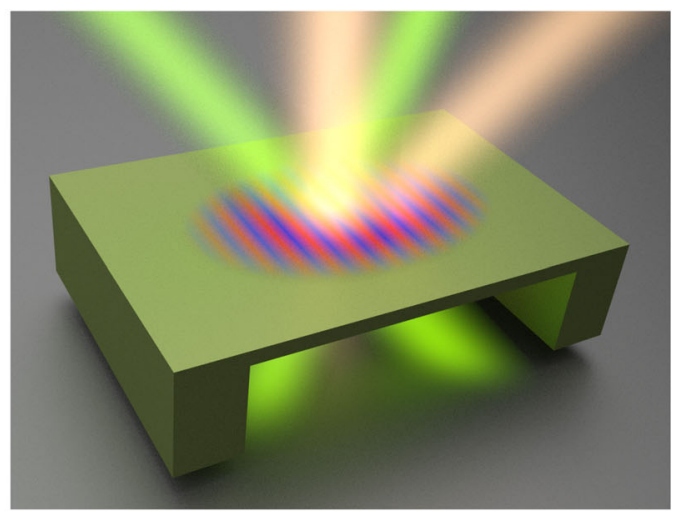

$$
\kappa(q)=\sum_{\zeta} S\left(q \Lambda_{\zeta}, \Lambda_{\zeta} / d, p_{\lambda}\right)\left[\frac{1}{3} C_{\zeta} v_{\zeta} \Lambda_{\zeta}\right] .
$$

In Eq. (1), $d$ is the thickness of the membrane, $\Lambda_{\zeta}$ is the mean free path, $C_{\zeta}$ is the volumetric mode-specific heat, and $v_{\zeta}$ is the group velocity of a phonon mode $\zeta \sim(\mathbf{k}, j)$ denoting a particular phonon with wave vector $\mathbf{k}$, polarization $j$, and wavelength $\lambda=2 \pi /|\mathbf{k}|$. In this theory, $p_{\lambda}$ is defined to be the directionally averaged, wavelengthdependent specularity parameter. A semianalytical expression for $S\left(q \Lambda_{\zeta}, \Lambda_{\zeta} / d, p_{\lambda}\right)$ for the experimental geometry in this work has been derived previously in Ref. [47], by solving the Boltzmann transport equation (BTE) for the transient decay of the initial sinusoidal temperature profile due to phonon transport.

The apparent thermal conductivity is thus a functional of the unknown specularity parameter versus wavelength $\left(p_{\lambda}\right)$. In our experiment, we vary the grating period, altering the suppression function $S\left(q \Lambda_{\zeta}, \Lambda_{\zeta} / d, p_{\lambda}\right)$, and the temperature, altering the phonon occupation, in effect filtering the phonon spectrum that contributes to heat transport and thereby generating constraints on the possible spectral dependence of the specularity parameter. The dispersion and intrinsic anharmonic lifetimes of thermal phonons in silicon are obtained from first-principles calculations [48]. Therefore, the unknown function, the specularity parameter versus phonon wavelength, can be extracted from measurements of $\kappa(q, T)$ by inverting Eq. (1).

Performing this extraction robustly requires certain inputs and experimental approaches. First, both temperaturedependent and grating period-dependent thermalconductivity measurements are required to sufficiently constrain the unknown $p_{\lambda}$, as we show in Sec. III. Second, the data must be interpreted using exact

(b)

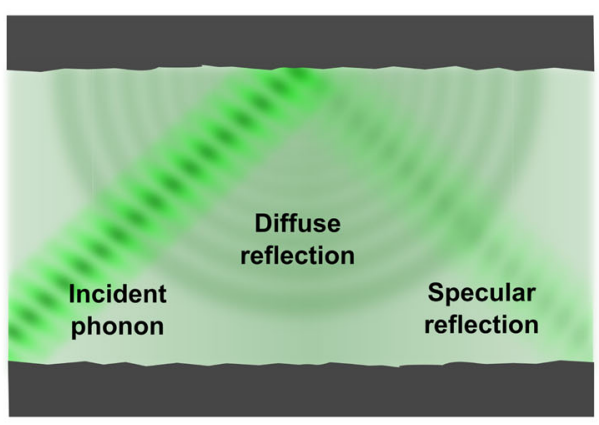

FIG. 1. (a) Schematic of the TG experiment. Two pump laser pulses (green) are crossed at the sample to form a sinusoidal temperature grating. A probe laser beam (bright orange) diffracts from the temperature grating and measures its transient decay. To improve the signal-to-noise ratio, the diffracted probe (signal) beam is optically heterodyned with a reference local oscillator beam (dull orange) and directed to a photodetector. (b) Excited thermal phonons reflect from the rough membrane boundaries either specularly or diffusely, depending on their wavelength. 
numerical solutions of the BTE under the relaxation-time approximation that incorporate partially specular phonon reflections [47] with first-principles phonon properties as input. This distinction is important, since the FuchsSondheimer theory is not applicable for phonons with MFPs longer than the TG grating period, as shown in our earlier work [47]. Data on thin Si membranes and the corresponding interpretation reported previously are thus insufficient to extract $p_{\lambda}$ [29].

We emphasize that our approach to obtain $p_{\lambda}$ does not require any knowledge of the surface roughness or details of the surface morphology. We assume no functional form for the dependence of $p_{\lambda}$ on the surface morphology of the thin film; thus, we require no information about it beforehand to extract $p_{\lambda}$. However, such information can help interpret the measurements of $p_{\lambda}$, and, in Sec. V, we provide structural characterization to aid in this interpretation.

\section{EXPERIMENT}

We implement the above-described strategy by measuring the thermal conductivity of freestanding silicon membranes using the TG technique from 80 to $400 \mathrm{~K}$. The TG experimental setup [Fig. 1(a)] is similar to that described in Ref. [29]. Briefly, a pair of pump pulses, shown by green beams in Fig. 1(a) (wavelength, $532 \mathrm{~nm}$; pulse width, approximately $1 \mathrm{~ns}$; energy per pulse, approximately $2 \mu \mathrm{J}$; repetition rate, $1 \mathrm{kHz}$ ), is focused on the sample to create a sinusoidal heating pattern that leads to a temperature grating. A continuous-wave probe laser, shown by the bright orange beam in Fig. 1(a) (wavelength, $514 \mathrm{~nm}$; power, approximately $200 \mathrm{~mW}$, chopped at $20 \%$ duty cycle to avoid steady heating of the sample), diffracts from the thermal grating, thereby monitoring the temporal decay of the grating on the sample. To improve the signal-to-noise ratio, the diffracted probe beam (not shown) is optically heterodyned with a reference beam (local oscillator; power, approximately $200 \mu \mathrm{W}$, chopped at $20 \%$ duty cycle), shown by the other orange beam in Fig. 1(a); the heterodyned beam is measured and recorded using a $1-\mathrm{GHz}$ photodetector and a $2.5-\mathrm{GHz}$ oscilloscope. The pump pulses produce a peak temperature rise of $\lesssim 5 \mathrm{~K}$ on the sample, while the weaker probe beam produces a temperature rise of $\lesssim 2 \mathrm{mK}$. Since the reference beam is strongly attenuated, the grating produced by the interference of the reference and signal beams is orders of magnitude weaker than that produced by the pump pulses.

We fabricate three suspended silicon membrane samples from silicon-on-insulator (SOI) wafers (MEMS Engineering and Materials Inc.) using wet etching techniques. We first spin-coat the SOI chip with a protective layer (ProTEK B3 from Brewer Science Inc.) and open a window on the bottom handle silicon layer by mechanically removing some of the protective ProTEK B3 material. Next, we dip the SOI chip in a potassium hydroxide $(\mathrm{KOH})$ hot bath $(50 \%$ concentration by weight at $70^{\circ} \mathrm{C}$ ) until the etch window reaches the buried oxide (BOX) layer. Finally, we remove the ProTEK B3 layer by dipping the sample into a bath of ProTEK remover 100 (Brewer Science Inc.), use wet HF etch to remove the BOX layer, and release the remaining silicon device layer into a suspended freestanding single-crystal silicon membrane. We fabricate three membranes with thicknesses of 1145 (M1), 515 (M2), and $590 \mathrm{~nm}$ (M3) using this procedure.

\section{RESULTS}

We perform TG experiments on the freestanding membranes from 80 to $400 \mathrm{~K}$ at several grating periods. Representative time traces of the sample response to the impulsive heating for membrane $M 1$ are shown in Fig. 2(a). We fit the time traces to a biexponential decay model to extract the apparent thermal conductivity of the membrane at different grating periods as described in Ref. [29].
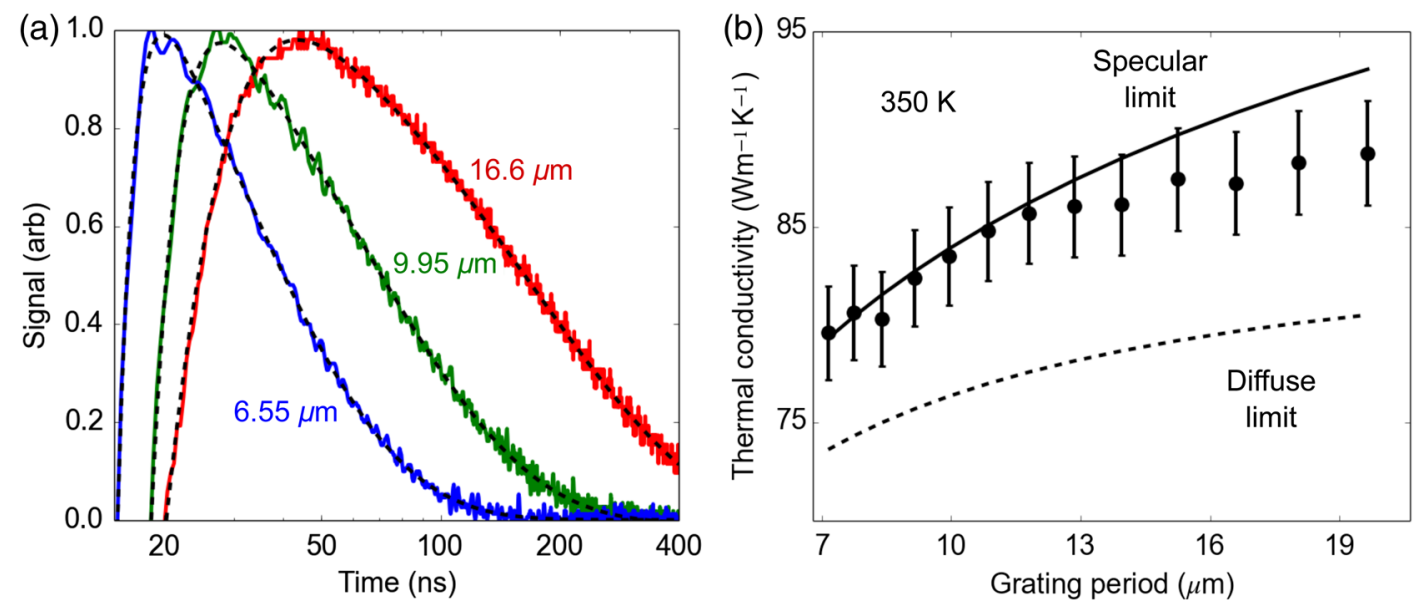

FIG. 2. (a) Representative transient signals from TG on thin silicon membranes at different grating periods and the corresponding biexponential fits (dashed black lines). (b) Measured thermal conductivity of membrane $M 1$ versus the grating period at $350 \mathrm{~K}$ obtained by fitting the data in (a). The thermal conductivity lies close to the specular limit. 
Figure 2(b) shows a representative measurement of apparent thermal conductivity versus the grating period along with the calculated specular and diffuse limits for thermal conductivity for membrane $M 1$ at $350 \mathrm{~K}$. The measured grating-period-dependent thermal conductivity of this membrane lies close to the specular limit at $350 \mathrm{~K}$, already indicating the occurrence of specular reflections of thermal phonons.

We begin analyzing the data at all temperatures and grating periods qualitatively by examining which functional forms of the specularity parameter are consistent with all of the data. Figure 3(a) shows three trial specularity parameter profiles versus the wavelength that have different functional dependencies on the phonon wavelength. Figures 3(b) and 3(c) show that only the monotonically increasing specularity profile, shown by the solid red curve in Fig. 3(a), captures the experimentally observed grating period and temperature dependence of the thermal conductivity simultaneously, while the other two profiles produce different trends compared to experiments.

The specific increasing profile in Fig. 3(a) is not the only one that can explain the data due to experimental uncertainty and the inherent ill-posedness of the inverse problem. Therefore, we generate a quantitative credible interval for the specularity parameter using Bayesian inference. Bayesian inference is a statistical method of solving inverse problems that rigorously combines the prior expected features of the specularity parameter with information from experiments. In this technique, we treat Eq. (1) as a model relating the specularity parameter to the data and minimize the objective function $\epsilon\left(p_{\lambda}\right)=\sum_{\delta, T}\left\|\kappa_{\text {expt }}-\kappa_{\text {model }}\left(p_{\lambda}\right)\right\|$ for random perturbations of the specularity parameter $\left(p_{\lambda}\right)$ from the solid red line in Fig. 3(a). The sum runs over the grating periods $\delta$ and temperatures $T$ used in the experiments. We choose a prior distribution function $\pi_{p_{\lambda}}$ that favors smoothness of the random perturbation. To collect statistics for Bayesian inference, we draw several trial specularity parameters $p_{\lambda}^{t}$ from the prior distribution function $\left(\pi_{p_{2}^{t}}\right)$ using a Metropolis Hastings Markov chain Monte Carlo (MHMCMC) method [49]. Finally, we invoke Bayes' theorem to compute the posterior probability distribution for each specularity parameter sample $p_{\lambda}^{t}$ using the error $\epsilon\left(p_{\lambda}^{t}\right)$ and the prior $\pi_{p_{\lambda}^{t}}$ as

$$
\pi_{\text {post }}\left(p_{\lambda}^{t} \mid \kappa^{\text {expt }}\right) \propto \mathcal{N}\left[\epsilon\left(p_{\lambda}^{t}\right), \sigma^{2} I\right] \times \pi_{\text {prior }}\left(p_{\lambda}^{t}\right) .
$$

In this equation, $\mathcal{N}\left[\epsilon\left(p_{\lambda}^{t}\right), \sigma^{2} I\right]$ represents the normal distribution function for the difference between our measurements and the prediction from the BTE solution for a trial specularity parameter, $p_{\lambda}^{t}$, with an experimental error $\sigma$ obtained from the measurements of the grating-perioddependent thermal conductivity in the TG experiment. The result of Bayesian inference is a posterior probability density $\left[\pi_{\text {post }}\left(p_{\lambda}^{t} \mid \kappa^{\text {expt }}\right)\right]$, which is proportional to the product of the prior probability distribution $\pi_{\text {prior }}\left(p_{\lambda}^{t}\right)$ and the extent to which $p_{\lambda}^{t}$ explains our measurements, given by $\mathcal{N}\left[\epsilon\left(p_{\lambda}^{t}\right), \sigma^{2} I\right]$. Intuitively, Eq. (2) states that a trial specularity profile $p_{\lambda}^{t}$ has a higher posterior probability density if it is a smooth function of the phonon wavelength and if the BTE solution with $p_{\lambda}^{t}$ matches well with the experimental data.

To start the Bayesian inference scheme, we choose an initial guess $p_{0}$ that best represents the experimental data points by trial and error. This process reduces the search space for possible specularity profiles that fit the measurements, thereby reducing the computational load. We evaluate Eq. (2) for more than $10^{5}$ trial specularity profiles drawn from the prior distribution $\left(\pi_{\text {prior }}\right)$ to obtain a converged posterior probability distribution $\left[\pi_{\text {post }}\left(p_{\lambda}\right)\right]$ for the phonon-wavelength-dependent specularity parameter $p_{\lambda}$ (see Supplemental Material, Sec. II [49], for details on the MHMCMC algorithm and the convergence of the Bayesian inference calculations). Thus, we are able to invert Eq. (1) using Bayesian inference to extract the phonon-wavelength-dependent specularity parameter from (a)

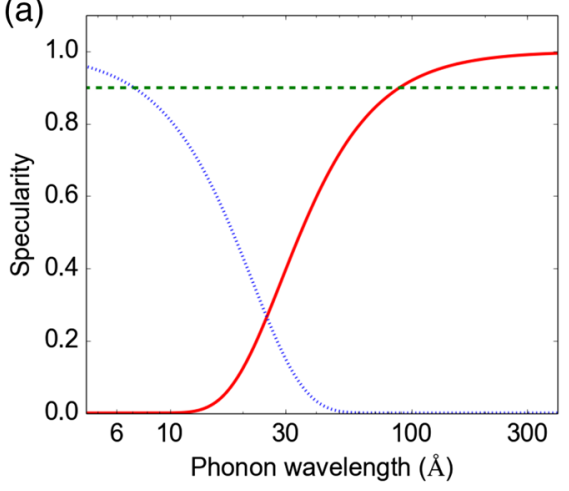

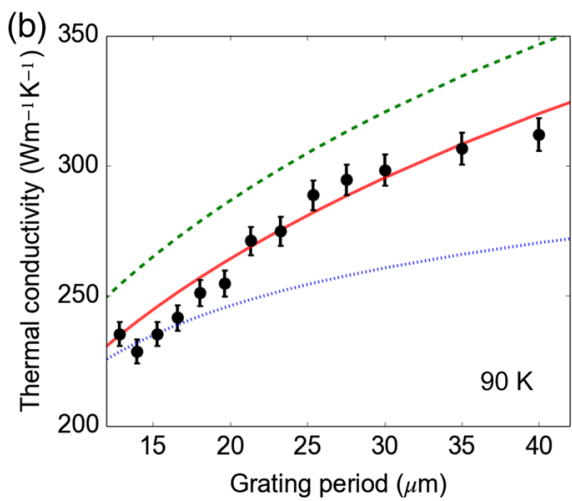

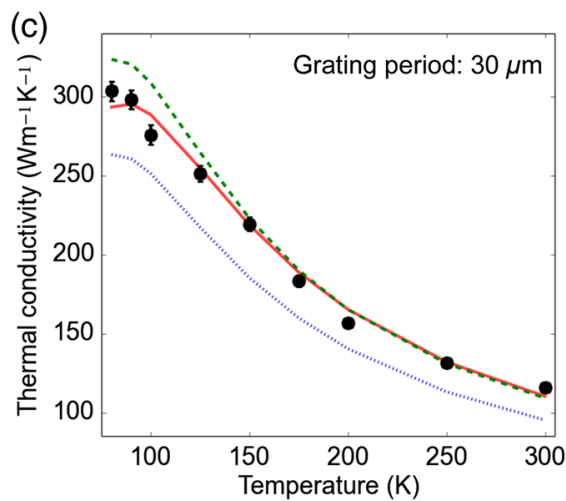

FIG. 3. (a) Candidate specularity parameters versus the phonon wavelength. (b) Thermal conductivity versus grating period at $90 \mathrm{~K}$ from the experiment (symbols) and the profiles in (a) (lines). (c) Thermal conductivity versus temperature for a grating period of $30 \mu \mathrm{m}$ from the experiment (symbols) and the profiles in (a) (lines). Only the increasing profile (red solid curve) matches all of the data. 
TG measurements of temperature-dependent and gratingperiod-dependent thermal conductivity.

The measured thermal conductivities versus the grating period at various temperatures along with those predicted by the posterior distribution of the specularity profile for membrane $M 1$ are given in Figs. 4(a)-4(d). The resulting posterior probability density for the specularity parameter versus the phonon wavelength is shown in Fig. 4(e). The gray region is an intensity plot of the posterior probability distribution for the specularity parameter, with the dashed lines indicating a 95\% credible interval. The extracted profile of the specularity parameter indicates that phonons with wavelengths less than approximately $20 \AA$ are nearly entirely diffusely reflected, while phonons with wavelengths longer than approximately $60 \AA$ are reflected predominantly specularly, thus quantitatively demonstrating partially specular reflections of thermal phonons $(\lambda \lesssim 100 \AA)$. The bounds for the specularity parameter in Fig. 4(e) are tighter than could be extracted from the thickness-dependent thin-film thermal-conductivity measurements reported in the literature [25-27,30-36] even after using $a b$ initio bulk phonon properties in the solution of the BTE to interpret their data (see Supplemental Material, Sec. IV [49], for details). A result similar to Fig. 4(e) is obtained on another sample with a thickness of $590 \mathrm{~nm}$ (see Supplemental Material, Sec. III [49]).

An examination of Figs. 4(a)-4(d) reveals several trends that are predicted by the specularity parameter presented in Fig. 4(e) and indirectly highlight the importance of including measurements at both different temperatures and grating periods to extract $p_{\lambda}$. First, observe that the apparent thermal conductivity in Figs. 4(a) and 4(b) is farther from the specular limit than at higher temperatures [Figs. 4(c) and 4(d)]. This result can be explained by the following argument. A phonon with a short intrinsic MFP, which is not affected by boundary reflections, has identical contributions to the specular and diffuse thermalconductivity limits in Figs. 4(d). Therefore, the TG experiment cannot distinguish between specular and diffuse boundary reflections of that phonon and is not sensitive to its specularity parameter. At $400 \mathrm{~K}$, almost all phonons with wavelengths shorter than approximately $30 \AA$ have intrinsic MFPs shorter than a tenth of the thickness of membrane $M 1$, so they rarely interact with the boundary. However, at $80 \mathrm{~K}$, the intrinsic MFPs of these phonons become comparable to the membrane thickness and reflect from the membrane boundary. Thus, the TG experiment is most sensitive to the specularity parameter of phonons of wavelengths $\lesssim 30 \AA$ only at low temperatures below around $100 \mathrm{~K}$.

On the other hand, measurements at room temperature and above are required to resolve the specularity parameter of long-wavelength phonons $(\lambda \gtrsim 30 \AA)$, since their thermal conductivity contributions below $100 \mathrm{~K}$ are quasiballistically suppressed owing to their long intrinsic MFPs compared to the TG grating periods [37]. Thus, performing grating-period-dependent measurements at just one temperature, as in Ref. [29], cannot provide enough sensitivity to
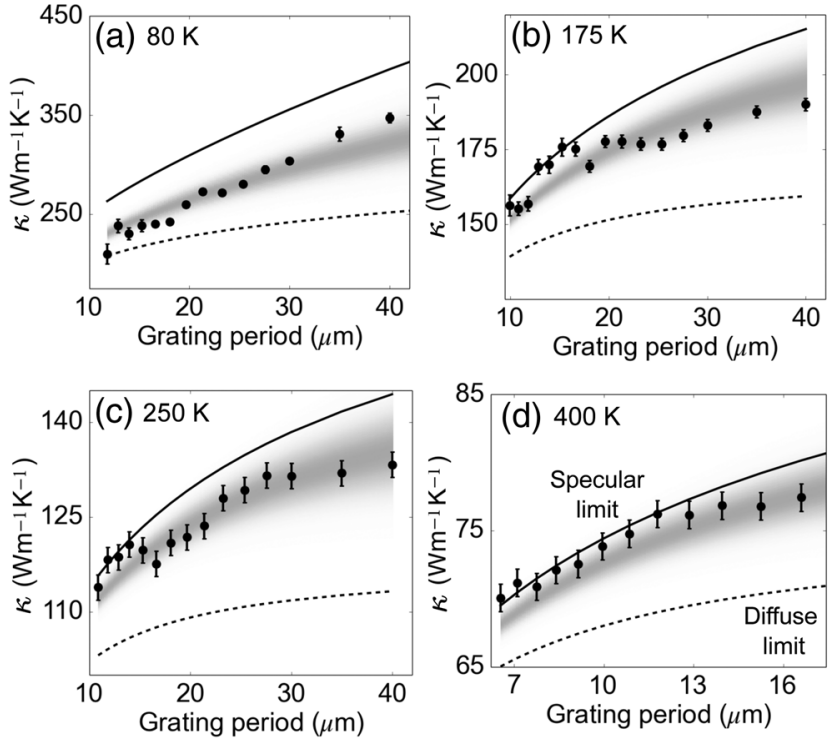

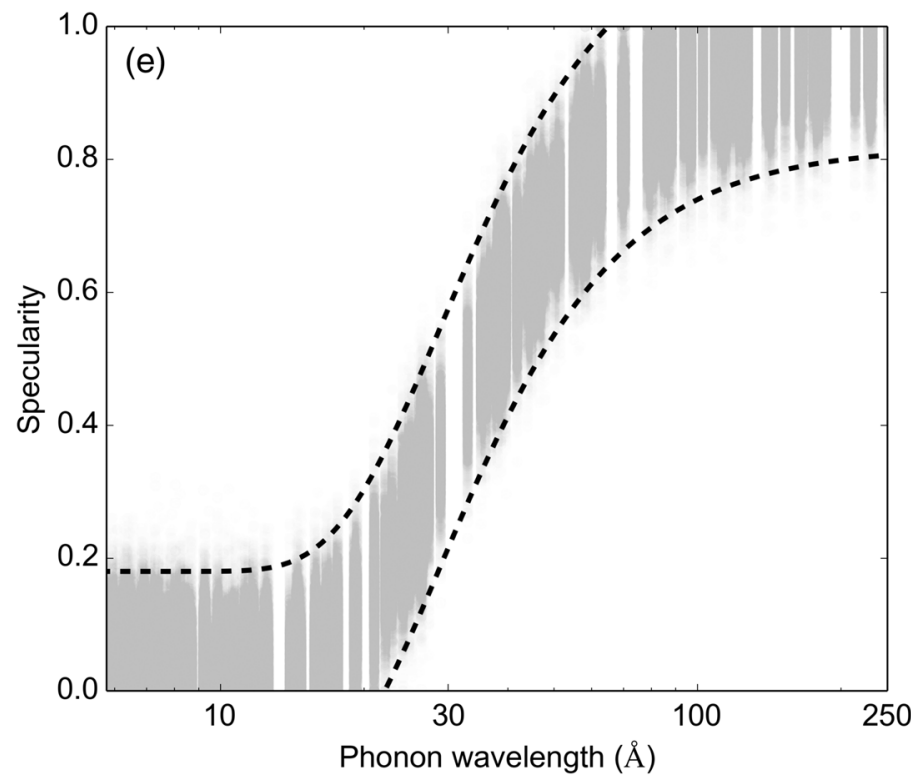

FIG. 4. Measured thermal conductivity of membrane $M 1$ at different grating periods for (a) 80, (b) 175, (c) 250, and (d) 400 K, compared with specular and diffuse limits. The gray region represents the thermal conductivity corresponding to the specularity parameter in (e). (e) Specularity parameter versus phonon wavelength obtained from Bayesian inference. The gray region is an intensity plot of the posterior probability distribution for the specularity parameter, with the dashed lines indicating a $95 \%$ credible interval. Phonons with wavelengths less than $20 \AA$ are nearly entirely diffusely reflected, while phonons with wavelengths longer than $60 \AA$ are reflected nearly completely specularly. 
constrain the specularity parameter versus the phonon wavelength.

The variation in sensitivity to different parts of the phonon spectrum is also evident in the thermal-conductivity measurements in Figs. 4(a)-4(d). At $400 \mathrm{~K}$, since shortwavelength phonons $(\lambda \lesssim 30 \AA)$ rarely interact with the membrane boundary and long-wavelength phonons $(\lambda \gtrsim 30 \AA)$ undergo predominantly specular reflections, the observed thermal conductivity is close to the specular limit. On the other hand, at temperatures below $100 \mathrm{~K}$, the sensitivity increases for short-wavelength phonons $(\lambda \lesssim$ $30 \AA$ ), which undergo partially specular reflections. Thus, the measured thermal conductivity is between the specular and diffuse limits. In fact, at $80 \mathrm{~K}$, we observe a transition from partially specular reflections at grating periods longer than approximately $20 \mu \mathrm{m}$ towards mostly diffuse reflections at short grating periods $(\lesssim 20 \mu \mathrm{m})$ in Fig. 4(a), due to quasiballistic suppression of even mid-to-short-wavelength ( $\lesssim 50 \AA$ ) phonons at short grating periods.

\section{DISCUSSION}

We obtain quantitative evidence that thermal phonons specularly reflect from the boundaries of Si membranes even around room temperature. Reaching this conclusion does not require any knowledge of the surface roughness of the membrane in our work. However, interpreting our results in the context of prior models for phonon reflections requires knowledge of the roughness, and we now seek to link our observations to the surface morphology of the membrane.

The most frequently used model to describe the specularity of phonon reflections is Ziman's model [13], and

(a) M1 - TEM

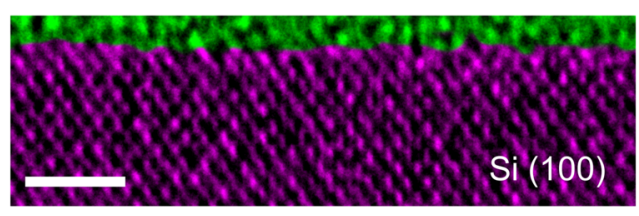

(c) M2 - TEM

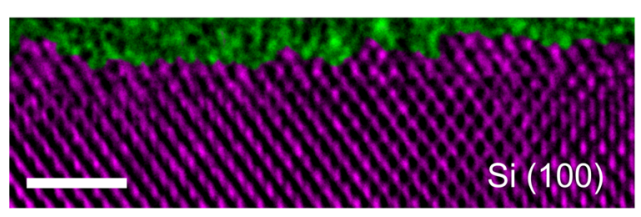

prior works draw conflicting conclusions on its validity for sub-THz phonons. For instance, Hertzberg et al. [24] report that sub-THz acoustic phonons reflect more diffusely than predicted by Ziman's model using the surface roughness of their samples obtained from atomic force microscopy (AFM), while Gelda et al. [22] report specular sub-THz phonon reflection consistent with Ziman's prediction with surface roughness obtained from transmission electron microscopy (TEM) characterization. Measurements for thermal phonons with frequencies around $10 \mathrm{THz}$ have not been reported previously.

In this work, we perform both AFM and TEM characterization of the membranes. For membrane $M 1$, crosssectional TEM imaging [Fig. 5(a)] yields a roughness of around $2.5 \pm 0.5 \AA$ corresponding to single atomic steps at the crystalline silicon-amorphous native-oxide surface, while AFM, which probes the free surface of the nativeoxide layer rather than the crystalline silicon-amorphous native-oxide interface, yields a roughness of $8 \pm 2 \AA$ [Fig. 5(b)]. These two limits for the roughness provide a large variation in the specularity parameter predicted by Ziman's theory: For a $50 \AA$ wavelength phonon, approximately $10 \AA \mathrm{rms}$ roughness results in completely diffuse reflection, while approximately $2 \AA \mathrm{rms}$ roughness predicts a specularity of 0.8 . Thus, a comparison with Ziman's theory for the entire thermal phonon spectrum probed is not so straightforward, since some phonons with wavelengths comparable to the atomic spacing likely reflect from the crystalline silicon-amorphous native-oxide interface, while longer-wavelength phonons may reflect from the free boundary with a different roughness than the crystalline silicon-amorphous native-oxide interface. (b) $M 1$ - AFM

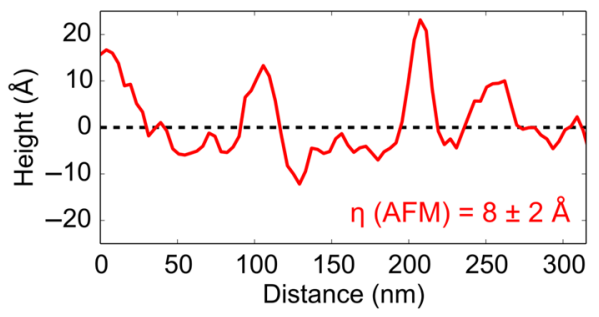

(d) M2 - AFM

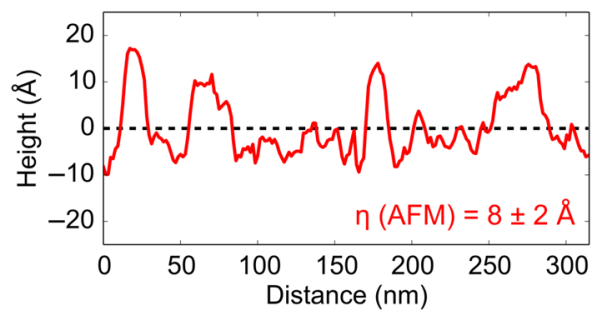

FIG. 5. Cross-sectional TEM images and AFM scans of the surface of membranes $M 1$ [(a) and (b), respectively] and $M 2$ [(c) and (d), respectively]. The rms roughness on the crystalline silicon membrane surface is estimated to be $2.5 \pm 0.5 \AA$ for membrane $M 1$ and $7 \pm 0.5 \AA$ for membrane M2. Scale bar, $20 \AA$. The AFM measurement on both membranes yielded a rms roughness of $8 \pm 2 \AA$. Additional TEM images on different locations of $M 1$ and $M 2$ are provided in Supplemental Material, Sec. I [49]. 


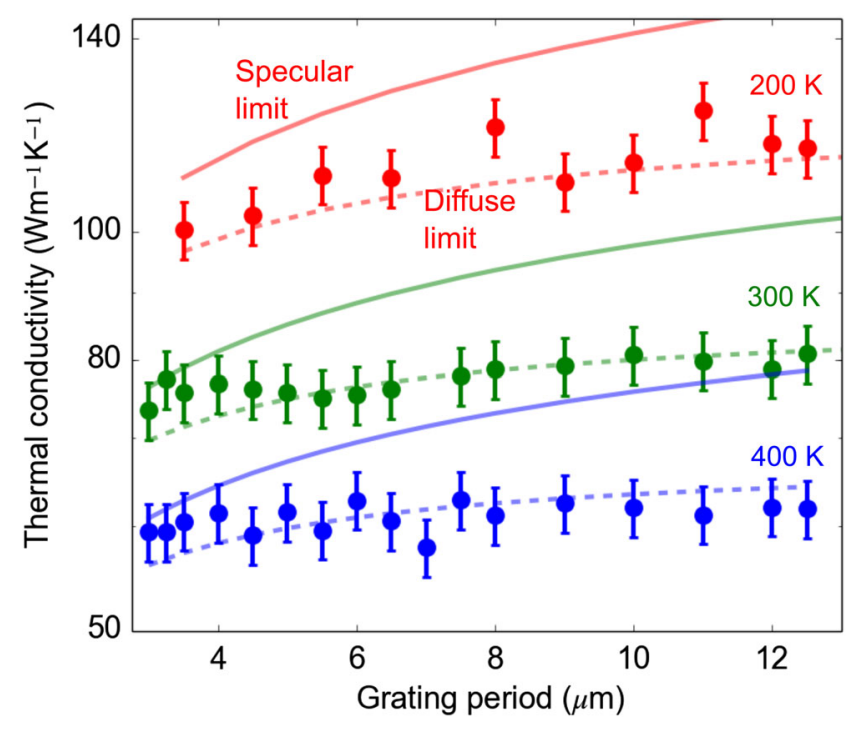

FIG. 6. Measured thermal conductivity of membrane M2 versus the grating period at various temperatures along with calculated specular and diffuse limits. The thermal conductivity lies nearly on the diffuse limit, indicating primarily diffuse reflections at the surface shown in Figs. 5(c) and 5(d).
We therefore cannot draw any conclusions about the validity of Ziman's model from our data. However, we can gain insight into which interface phonons reflect from and how these reflections are influenced by surface roughness. If some fraction of thermal phonons reflect from the crystalline silicon-native-oxide interface, then a membrane with similar roughness at both the crystalline silicon-native-oxide and native-oxide-vacuum interfaces of around $10 \AA$ would be expected to exhibit thermal conductivity closer to the diffuse limit than $M 1$, since both interfaces would act as diffuse phonon reflectors. If all phonons reflect from the free nativeoxide boundary with the same roughness as $M 1$, the extracted specularity parameter of the second membrane should be unchanged compared to that of $M 1$.

We identified a membrane $(M 2)$ with a nominal thickness of $515 \mathrm{~nm}$ with rms roughness at the crystalline silicon-native-oxide interface as $7 \pm 0.5 \AA$ from images by TEM and rms roughness of $8 \pm 2 \AA$ from AFM [Figs. 5(c) and 5(d), respectively]. The thermal-conductivity measurements on this membrane are shown in Fig. 6 at various temperatures, and, as predicted, they lie close to the fully diffuse limit. Thus, our results confirm that at least some of
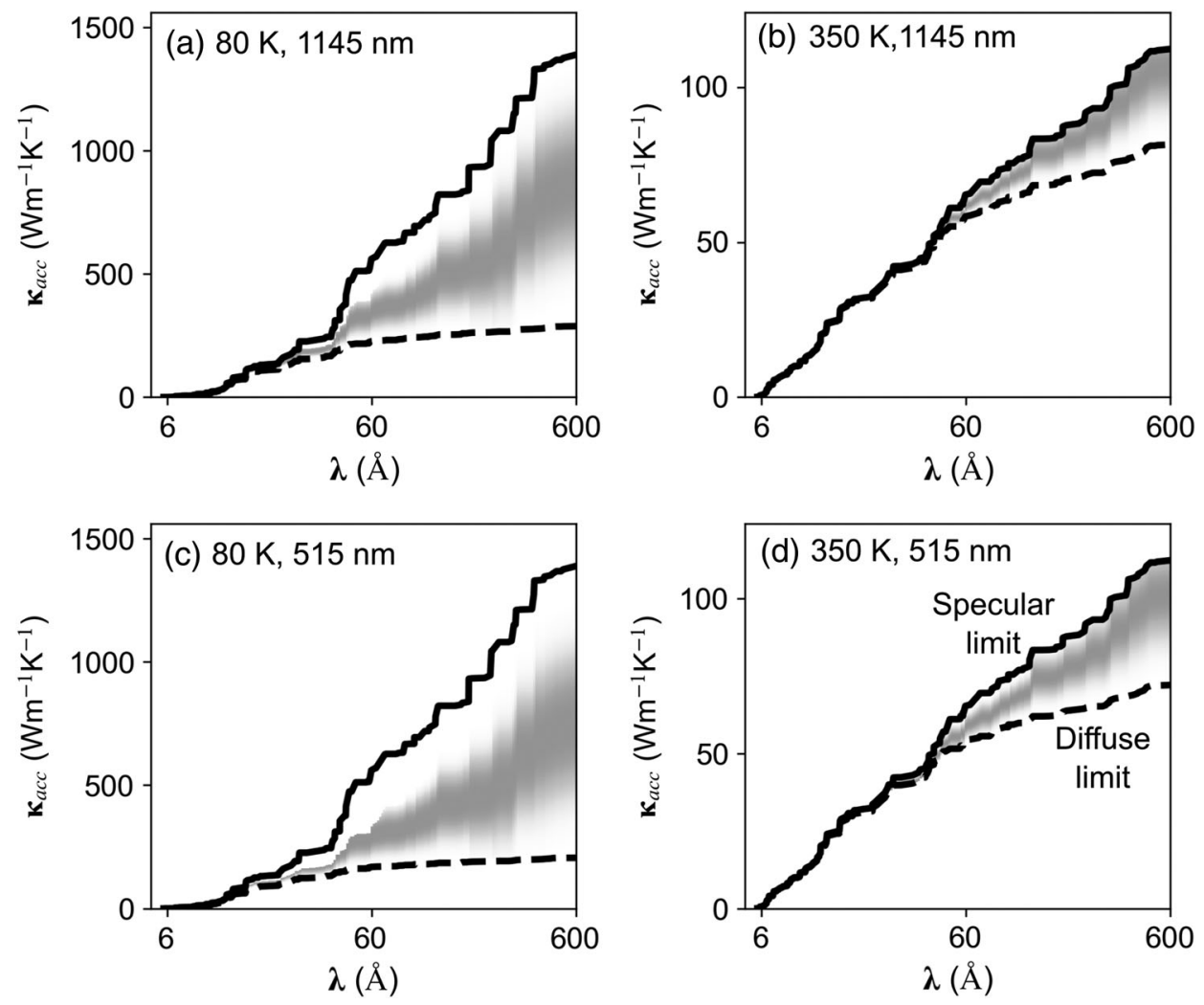

FIG. 7. Calculated thermal-conductivity accumulation versus phonon wavelength for membranes with thicknesses of $1145 \mathrm{~nm}$ [(a),(b)] corresponding to $M 1$ and $515 \mathrm{~nm}[(\mathrm{c}),(\mathrm{d})]$ corresponding to $M 2$ at 80 and $350 \mathrm{~K}$. The solid (dashed) black curve represents the specular (diffuse) scattering limit, and the gray region is calculated using the measured specularity profile in Fig. 4(e). Partially specular reflections as in Fig. 4(e) enhance the thermal conductivity of $M 1$ by $40 \%-500 \%$ at $80 \mathrm{~K}$ and $15 \%-50 \%$ at $350 \mathrm{~K}$ compared to the diffuse limit. Since $M 2$ is thinner, a specularity profile as in Fig. 4(e) could enhance the thermal conductivity by $100 \%-600 \%$ at $80 \mathrm{~K}$ and $20 \%-60 \%$ at $350 \mathrm{~K}$ compared to the observed diffuse limit. 
the thermal phonons with a frequency of around a few terahertz are reflected from the crystalline siliconnative-oxide interface and that they are sensitive to Angstrom-scale changes in the surface roughness (see Supplemental Material, Sec. II [49], for thermalconductivity measurements and the specularity parameter for a third membrane $M 3$ ).

The significance of observing partially specular thermal phonon reflections at atomically smooth surfaces of sample $M 1$ rather than fully diffuse reflections as in sample $M 2$ is elucidated in Fig. 7. Here, the thermal-conductivity accumulations versus the phonon wavelength are presented for the extracted specularity parameter of sample $M 1$ and for the diffuse limit observed in sample $M 2$, calculated $a b$ initio. Notice that the specular limit is independent of the film thickness at a given temperature, while the diffuse limit is lower for thinner films. Partially specular reflections of long-wavelength thermal phonons $(\lambda \gtrsim 30 \AA)$ as in Fig. 4(e) increase the total accumulated thermal conductivity of micron-thick films by at least $40 \%$ and as much as $500 \%$ from the diffuse limit at $80 \mathrm{~K}$, as shown in Fig. 7(a). The enhancement is weaker at higher temperatures [at least $15 \%$ at $350 \mathrm{~K}$, as shown in Fig. 7(b)], where phonons with wavelengths less than approximately $50 \AA$ have shorter MFPs than the film thickness and are unaffected by surface reflections, as shown by the overlapping specular and diffuse limits up to approximately $50 \AA$ in Figs. 7(b) and 7(d). Furthermore, the influence of partially diffuse surface reflections of phonons on thermal conductivity is larger in thinner films [Figs. 7(c) and 7(d)], resulting in a lower total accumulated thermal conductivity than the thicker films. Thus, our approach enables the determination of precisely which phonons likely undergo specular reflections and what is the resulting impact on the thermal conductivity, which is not possible using available data in the literature [25-27,30-36] (see Supplemental Material, Sec. IV [49], for details).

These observations also have important implications for recent efforts to create novel materials by interfering thermal phonons [9-11], as specular reflection is a necessary requirement for coherent interference. Our results suggest that, although lithographically etched surfaces are unlikely to be sufficiently smooth for specular reflections at room temperature, the required conditions can indeed be met with epitaxially grown films with atomically smooth surfaces, providing a route forward for coherent manipulation of heat.

\section{SUMMARY}

In summary, we have reported the measurements of the specularity parameter as a spectral function of the phonon wavelength in atomically rough silicon membranes using optical TG experiments and $a b$ initio analysis of the experimental data. Our measurements show that thermal phonons can specularly reflect from high-quality wafer surfaces even around room temperature and that these reflections are sensitive to imperfections on the order of a few atomic planes. Additionally, our work demonstrates a general approach to probe the microscopic processes governing thermal phonon-boundary interactions by interpreting experimental observables using the Boltzmann transport equation with $a b$ initio phonon properties as input.

\section{ACKNOWLEDGMENTS}

This work was supported by the National Science Foundation under Grant No. CBET CAREER 1254213. The authors thank A. A. Maznev and K. A. Nelson for fruitful discussions about the TG experiment and $\mathrm{L}$. Lindsay for providing the $a b$ initio phonon properties. N.K.R. thanks the Resnick Sustainability Institute at Caltech and the Dow Chemical Company for fellowship support.

A. J. M. and N. K. R. originated the research. H. Z. and N. K. R. fabricated the silicon membrane samples. N. K. R. conducted the TG experiments and performed the BTE and the Bayesian inference calculations. N.K. R. performed the TEM imaging with help from Carol Garland at the Kavli Nanoscience Institute at Caltech. N. K. R. and A. J. M. analyzed the results and prepared the manuscript. All authors studied and commented on the manuscript.

The authors declare no competing financial interests.

[1] P. Beckmann and A. Spizzichino, The Scattering of Electromagnetic Waves from Rough Surfaces, Radar Library (Artech House, Washington, DC, 1987).

[2] J. G. Watson and J. B. Keller, Reflection, Scattering, and Absorption of Acoustic Waves by Rough Surfaces, J. Acoust. Soc. Am. 74, 1887 (1983).

[3] C. Chapman, Fundamentals of Seismic Wave Propagation (Cambridge University Press, Cambridge, England, 2004).

[4] D. G. Cahill, P. V. Braun, G. Chen, D. R. Clarke, S. Fan, K. E. Goodson, P. Keblinski, W. P. King, G. D. Mahan, A. Majumdar, H. J. Maris, S. R. Phillpot, E. Pop, and L. Shi, Nanoscale Thermal Transport. II, Appl. Phys. Rev. 1, 011305 (2014).

[5] R. Chen, A. I. Hochbaum, P. Murphy, J. Moore, P. Yang, and A. Majumdar, Thermal Conductance of Thin Silicon Nanowires, Phys. Rev. Lett. 101, 105501 (2008).

[6] K. Hippalgaonkar, B. Huang, R. Chen, K. Sawyer, P. Ercius, and A. Majumdar, Fabrication of Microdevices with Integrated Nanowires for Investigating Low-Dimensional Phonon Transport, Nano Lett. 10, 4341 (2010).

[7] J. Tang, H.-T. Wang, D. H. Lee, M. Fardy, Z. Huo, T. P. Russell, and P. Yang, Holey Silicon as an Efficient Thermoelectric Material, Nano Lett. 10, 4279 (2010).

[8] J. Lee, W. Lee, G. Wehmeyer, S. Dhuey, D. L. Olynick, S. Cabrini, C. Dames, J. J. Urban, and P. Yang, Investigation of 
Phonon Coherence and Backscattering Using Silicon Nanomeshes, Nat. Commun. 8, 14054 (2017).

[9] M. N. Luckyanova, J. Garg, K. Esfarjani, A. Jandl, M. T. Bulsara, A. J. Schmidt, A. J. Minnich, S. Chen, M. S. Dresselhaus, Z. Ren, E. A. Fitzgerald, and G. Chen, Coherent Phonon Heat Conduction in Superlattices, Science 338, 936 (2012).

[10] J. Ravichandran, A. K. Yadav, R. Cheaito, P. B. Rossen, A. Soukiassian, S. J. Suresha, J. C. Duda, B. M. Foley, C.-H. Lee, Y. Zhu, A. W. Lichtenberger, J. E. Moore, D. A. Muller, D. G. Schlom, P. E. Hopkins, A. Majumdar, R. Ramesh, and M. A. Zurbuchen, Crossover from Incoherent to Coherent Phonon Scattering in Epitaxial Oxide Superlattices, Nat. Mater. 13, 168 (2014).

[11] J. Maire, R. Anufriev, R. Yanagisawa, A. Ramiere, S. Volz, and M. Nomura, Heat Conduction Tuning by Wave Nature of Phonons, Sci. Adv. 3, e1700027 (2017).

[12] M. Maldovan, Sound and Heat Revolutions in Phononics, Nature (London) 503, 209 (2013).

[13] J. M. Ziman, Electrons and Phonons: The Theory of Transport Phenomena in Solids (Oxford University, New York, 1960).

[14] A. A. Maznev, Boundary Scattering of Phonons: Specularity of a Randomly Rough Surface in the Small-Perturbation Limit, Phys. Rev. B 91, 134306 (2015).

[15] J. Carrete, L. J. Gallego, L. M. Varela, and N. Mingo, Surface Roughness and Thermal Conductivity of Semiconductor Nanowires: Going below the Casimir Limit, Phys. Rev. B 84, 075403 (2011).

[16] J. Sadhu and S. Sinha, Room-Temperature Phonon Boundary Scattering below the Casimir Limit, Phys. Rev. B 84, 115450 (2011).

[17] Z. Liang, K. Sasikumar, and P. Keblinski, Thermal Transport across a Substrate-Thin Film Interface: Effects of Film Thickness and Surface Roughness, Phys. Rev. Lett. 113, 065901 (2014).

[18] L. N. Maurer, S. Mei, and I. Knezevic, Rayleigh Waves, Surface Disorder, and Phonon Localization in Nanostructures, Phys. Rev. B 94, 045312 (2016).

[19] Z. Wei, Y. Chen, and C. Dames, Wave Packet Simulations of Phonon Boundary Scattering at Graphene Edges, J. Appl. Phys. 112, 024328 (2012).

[20] E. T. Swartz and R. O. Pohl, in Phonon Scattering in Condensed Matter V, Springer Series in Solid-State Sciences Vol. 68, edited by A. C. Anderson and J. P. Wolfe (Springer, Berlin, 1986), pp. 228-230.

[21] T. Klitsner, J. E. VanCleve, H. E. Fischer, and R. O. Pohl, Phonon Radiative Heat Transfer and Surface Scattering, Phys. Rev. B 38, 7576 (1988).

[22] D. Gelda, M. G. Ghossoub, K. Valavala, J. Ma, M. C. Rajagopal, and S. Sinha, Specularity of Longitudinal Acoustic Phonons at Rough Surfaces, Phys. Rev. B 97, 045429 (2018).

[23] J. S. Heron, T. Fournier, N. Mingo, and O. Bourgeois, Mesoscopic Size Effects on the Thermal Conductance of Silicon Nanowire, Nano Lett. 9, 1861 (2009).

[24] J. B. Hertzberg, M. Aksit, O. O. Otelaja, D. A. Stewart, and R. D. Robinson, Direct Measurements of Surface Scattering in Si Nanosheets Using a Microscale Phonon Spectrometer:
Implications for Casimir-Limit Predicted by Ziman Theory, Nano Lett. 14, 403 (2014).

[25] M. Asheghi, Y. K. Leung, S. S. Wong, and K. E. Goodson, Phonon-Boundary Scattering in Thin Silicon Layers, Appl. Phys. Lett. 71, 1798 (1997).

[26] Y. S. Ju and K. E. Goodson, Phonon Scattering in Silicon Films with Thickness of Order $100 \mathrm{~nm}$, Appl. Phys. Lett. 74, 3005 (1999).

[27] W. Liu and M. Asheghi, Thermal Conduction in Ultrathin Pure and Doped Single-Crystal Silicon Layers at High Temperatures, J. Appl. Phys. 98, 123523 (2005).

[28] J. P. Feser, J. S. Sadhu, B. P. Azeredo, K. H. Hsu, J. Ma, J. Kim, M. Seong, N. X. Fang, X. Li, P. M. Ferreira, S. Sinha, and D. G. Cahill, Thermal Conductivity of Silicon Nanowire Arrays with Controlled Roughness, J. Appl. Phys. 112, 114306 (2012).

[29] J. A. Johnson, A. A. Maznev, J. Cuffe, J. K. Eliason, A. J. Minnich, T. Kehoe, C. M. S. Torres, G. Chen, and K. A. Nelson, Direct Measurement of Room-Temperature Nondiffusive Thermal Transport over Micron Distances in a Silicon Membrane, Phys. Rev. Lett. 110, 025901 (2013).

[30] E. Chavez-Angel, J. S. Reparaz, J. Gomis-Bresco, M. R. Wagner, J. Cuffe, B. Graczykowski, A. Shchepetov, H. Jiang, M. Prunnila, J. Ahopelto, F. Alzina, and C. M. Sotomayor Torres, Reduction of the Thermal Conductivity in Free-Standing Silicon Nano-Membranes Investigated by Non-Invasive Raman Thermometry, APL Mater. 2, 012113 (2014).

[31] J. Cuffe, J. K. Eliason, A. A. Maznev, K. C. Collins, J. A. Johnson, A. Shchepetov, M. Prunnila, J. Ahopelto, C. M. S. Torres, G. Chen, and K. A. Nelson, Reconstructing Phonon Mean-Free-Path Contributions to Thermal Conductivity Using Nanoscale Membranes, Phys. Rev. B 91, 245423 (2015).

[32] M. Asheghi, M. N. Touzelbaev, K. E. Goodson, Y. K. Leung, and S. S. Wong, Temperature-Dependent Thermal Conductivity of Single-Crystal Silicon Layers in SOI Substrates, J. Heat Transfer 120, 30 (1998).

[33] Z. Hao, L. Zhichao, T. Lilin, T. Zhimin, L. Litian, and L. Zhijian, in Proceedings of the 8th International Conference on Solid-State and Integrated Circuit Technology, Shanghai, 2006 (IEEE, 2006), pp. 2196-2198, https://ieeexplore .ieee.org/document/4098666.

[34] W. Liu and M. Asheghi, Thermal Conductivity Measurements of Ultra-Thin Single Crystal Silicon Layers, J. Heat Transfer 128, 75 (2006).

[35] M. S. Aubain and P. R. Bandaru, Determination of Diminished Thermal Conductivity in Silicon Thin Films Using Scanning Thermoreflectance Thermometry, Appl. Phys. Lett. 97, 253102 (2010).

[36] M. S. Aubain and P. R. Bandaru, In-Plane Thermal Conductivity Determination through Thermoreflectance Analysis and Measurements, J. Appl. Phys. 110, 084313 (2011).

[37] A. J. Minnich, Determining Phonon Mean Free Paths from Observations of Quasiballistic Thermal Transport, Phys. Rev. Lett. 109, 205901 (2012).

[38] C. Hua, X. Chen, N. K. Ravichandran, and A. J. Minnich, Experimental Metrology to Obtain Thermal Phonon Transmission Coefficients at Solid Interfaces, Phys. Rev. B 95, 205423 (2017). 
[39] K. T. Regner, A. J. H. McGaughey, and J. A. Malen, Analytical Interpretation of Nondiffusive Phonon Transport in Thermoreflectance Thermal Conductivity Measurements, Phys. Rev. B 90, 064302 (2014).

[40] K. M. Hoogeboom-Pot, J. N. Hernandez-Charpak, X. Gu, T. D. Frazer, E. H. Anderson, W. Chao, R. W. Falcone, R. Yang, M. M. Murnane, H. C. Kapteyn, and D. Nardi, A New Regime of Nanoscale Thermal Transport: Collective Diffusion Increases Dissipation Efficiency, Proc. Natl. Acad. Sci. U.S.A. 112, 4846 (2015).

[41] C. Jeong, S. Datta, and M. Lundstrom, Thermal Conductivity of Bulk and Thin-Film Silicon: A Landauer Approach, J. Appl. Phys. 111, 093708 (2012).

[42] A. T. Ramu and J. E. Bowers, On the Solenoidal Heat-Flux in Quasi-Ballistic Thermal Conduction, J. Appl. Phys. 118, 125106 (2015).

[43] M. E. Siemens, Q. Li, R. Yang, K. A. Nelson, E. H. Anderson, M. M. Murnane, and H.C. Kapteyn, QuasiBallistic Thermal Transport from Nanoscale Interfaces Observed Using Ultrafast Coherent Soft X-Ray Beams, Nat. Mater. 9, 26 (2010).

[44] A. J. Minnich, J. A. Johnson, A. J. Schmidt, K. Esfarjani, M. S. Dresselhaus, K. A. Nelson, and G. Chen, Thermal
Conductivity Spectroscopy Technique to Measure Phonon Mean Free Paths, Phys. Rev. Lett. 107, 095901 (2011).

[45] V. Chiloyan, L. Zeng, S. Huberman, A. A. Maznev, K. A. Nelson, and G. Chen, Variational Approach to Solving the Spectral Boltzmann Transport Equation in Transient Thermal Grating for Thin Films, J. Appl. Phys. 120, 025103 (2016).

[46] C. Hua and A. J. Minnich, Transport Regimes in Quasiballistic Heat Conduction, Phys. Rev. B 89, 094302 (2014).

[47] N. K. Ravichandran and A. J. Minnich, Role of Thermalizing and Nonthermalizing Walls in Phonon Heat Conduction along Thin Films, Phys. Rev. B 93, 035314 (2016).

[48] L. Lindsay, D. A. Broido, and T. L. Reinecke, Ab Initio Thermal Transport in Compound Semiconductors, Phys. Rev. B 87, 165201 (2013).

[49] See Supplemental Material at http://link.aps.org/ supplemental/10.1103/PhysRevX.8.041004 for (i) additional TEM images for membranes $M 1$ and $M 2$, (ii) additional details on the $a b$ initio procedure to extract specularity parameter, (iii) results for membrane $M 3$ (590 nm thick), and (iv) an analysis of prior works with $a b$ initio properties. 